\title{
Is an "Aerosol Box" Needed in Patients Planned to Undergo Rapid Sequence Induction Before Intubation in the Operating Room?
}

\author{
Yusuke Iizuka ${ }^{\mathrm{a}, \mathrm{c}}$, Yuji Otsuka ${ }^{\mathrm{a}}$, Takeshi Nakatomi ${ }^{\mathrm{a}}$, Koichi Yoshinaga ${ }^{\mathrm{a}}$, \\ Alan Kawarai Lefor ${ }^{\mathrm{b}}$, Masamitsu Sanui ${ }^{\mathrm{a}}$
}

\section{To the Editor}

During the coronavirus disease 2019 (COVID-19) outbreak, efforts to minimize healthcare workers' exposure to aerosols have expanded. In the operating room, many patients suspected to have or infected with COVID-19 present for surgery. To prevent aerosol exposure, rapid sequence induction (RSI) after adequate preoxygenation with a tight-fitting mask and use of a video laryngoscope are recommended [1]. During these procedures, anesthesiologists and other operating room staff are faced with potential contamination; droplets are created by coughing and aerosolized particles leak from the mask. Recently, an "aerosol box" was introduced [2] and is widely used in intensive care units and operating rooms. The aerosol box is useful to prevent direct droplet exposure during awake intubation, in which coughing is inevitable. It is also used for patients with severe hypoxemia or who are anticipated to have a difficult airway. However, in the operating room, most patients undergo RSI and do not cough after adequate muscle relaxation. Conventional operating rooms have ventilation systems to produce a minimum of 15 -air changes per hour [3]. Even if aerosolized particles leak from the mask during preoxygenation, filtered air from the ceiling to the exhaust duct should remove them immediately without an aerosol box. Using an aerosol box can retain the aerosolized particles in the box due to interruption of ventilation. The importance of the aerosol box in the operating room is unclear.

To visualize aerosolized particles with or without an aerosol box in the operating room, a simulation model with a mannequin connected to an ultrasound nebulizer (UN-511, Alfresa, Osaka, Japan) generating mist was used. The level of

Manuscript submitted July 21, 2020, accepted July 23, 2020

Published online August 22, 2020

aDepartment of Anesthesiology and Critical Care Medicine, Jichi Medical University Saitama Medical Center, Saitama, Japan

bepartment of Surgery, Jichi Medical University, Tochigi, Japan

${ }^{\mathrm{c} C o r r e s p o n d i n g ~ A u t h o r: ~ Y u s u k e ~ I i z u k a, ~ D e p a r t m e n t ~ o f ~ A n e s t h e s i o l o g y ~ a n d ~}$ Critical Care Medicine, Jichi Medical University Saitama Medical Center, 1-847 Amanuma-cho, Omiya-ku, Saitama-shi, Saitama-ken 330-8503, Japan. Email: zukarinn@gmail.com

doi: https://doi.org/10.14740/jcs418 aerosolized particles was measured using a particulate matter $(<2.5 \mu \mathrm{m}$, PM2.5) detector (DM106, Dienmern, Shenzhen, China). The mannequin was set below the ceiling duct which supplies filtered air. The PM2.5 detector was placed near the mannequin's head. We simulated changing levels of aerosolized particles around the head of the mannequin with a facemask (aerosol leakage model during preoxygenation) with or without an aerosol box. We used two types of aerosol boxes. Aerosol box A is an acrylic cube with two circular ports (Fig. $1 \mathrm{a}, \mathrm{b})$ and box $\mathrm{B}$ is a semicircular metal frame with disposable drapes (Fig. 1c, d). Aerosol box B has two small slits allowing the anesthesiologist's hands to pass to a cranial drape and we covered the chest with a drape to increase airtightness. Aerosol box A was recently acquired, and we have been using aerosol box B clinically. Figure 2 shows resulting levels of aerosolized particles around the head of the mannequin with a facemask. Without an aerosol box, the generated mist was forced to flow to the floor. The level of PM2.5 was $42 \mu \mathrm{g} / \mathrm{m}^{3}$. With aerosol box A, downstream flow from the ceiling was interrupted, the

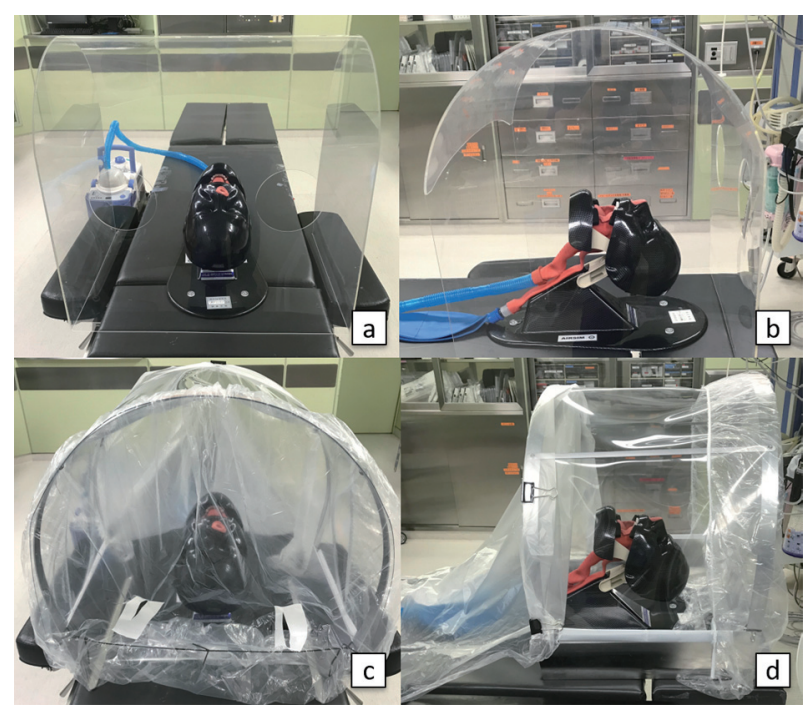

Figure 1. (a, b) Aerosol box A: an acrylic cube with two circular ports. (c, d) Aerosol box B: a semicircular metal frame with disposable drapes. Aerosol box $\mathrm{B}$ has two small slits to pass the anesthesiologist's hand to a cranial drape and the chest is covered with a caudal drape to increase airtightness. 


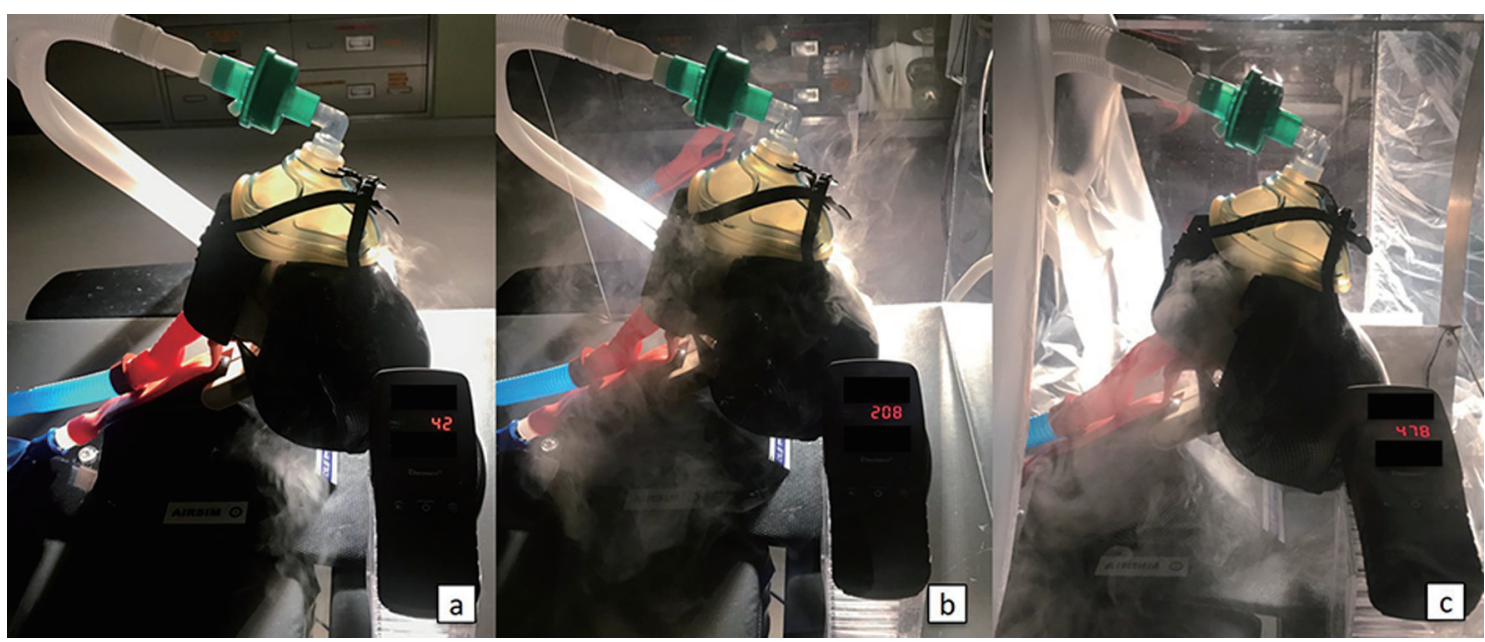

Figure 2. Levels of PM2.5. (a) PM2.5 detector showed $42 \mu \mathrm{g} / \mathrm{m}^{3}$ without an aerosol box. (b) PM2.5 detector showed $208 \mu \mathrm{g} / \mathrm{m}^{3}$ in aerosol box A. (c) PM2.5 detector showed $478 \mu \mathrm{g} / \mathrm{m}^{3}$ in aerosol box B.

generated mist was stored in the box, and the level of PM2.5 increased to $208 \mu \mathrm{g} / \mathrm{m}^{3}$. With aerosol box B, due to increased airtightness, the level of PM2.5 increased to $478 \mu \mathrm{g} / \mathrm{m}^{3}$. In all simulations, the level of PM2.5 around the anesthesiologist's head was $0 \mu \mathrm{g} / \mathrm{m}^{3}$.

This simulation shows that using an aerosol box during preoxygenation could increase the level of aerosolized particles in the box due to their airtightness. This simulation did not visualize the distribution or measure levels of particles smaller than $2.5 \mu \mathrm{m}$, which may cause air-borne infections, but the distribution of such smaller particles may have a similar trend as PM2.5 [4].

Aerosol boxes have several disadvantages. First, the aerosol box might restrict hand movements, and might be an additional contagious surface. In most patients planned to undergo RSI, a tight-fitting mask during preoxygenation is enough to prevent direct exposure to droplets. Second, using an aerosol box might increase the risk of aerosolized particle exposure. When an aerosol box is used during preoxygenation, ventilation is interrupted, and leaked aerosolized particles are stored in the box as this simulation showed. These results raise a serious concern that anesthesiologists might be exposed to high levels of infectious aerosolized particles just after removal of the aerosol box following preoxygenation.

An aerosol box might not be needed during preoxygenation in patients planned to undergo RSI. An aerosol box might be used just before intubation and may be unnecessary with adequate muscle relaxation in conventional operating rooms.

\section{Acknowledgments}

None to declare.

\section{Financial Disclosure}

None to declare.

\section{Conflict of Interest}

The authors declare that there is no conflict of interest.

\section{Informed Consent}

Not applicable.

\section{Author Contributions}

YI, YO, TN, and KY have given substantial contributions to the conception of the manuscript. All authors have participated to drafting the manuscript. YI, MS, and AL revised it critically. All authors read and approved the final version of the manuscript.

\section{Data Availability}

The data supporting the findings of this study are available from the corresponding author upon reasonable request.

\section{References}

1. Peng PWH, Ho PL, Hota SS. Outbreak of a new coronavirus: what anaesthetists should know. Br J Anaesth. 2020;124(5):497-501.

2. Canelli R, Connor CW, Gonzalez M, Nozari A, Ortega R. Barrier enclosure during endotracheal intubation. N Engl J Med. 2020;382(20):1957-1958.

3. Sehulster L, Chinn RY, Cdc Hicpac. Guidelines for environmental infection control in health-care facilities. Recommendations of CDC and the Healthcare Infection Control Practices Advisory Committee (HICPAC). MMWR 
Recomm Rep. 2003;52(RR-10):1-42.

4. Simpson JP, Wong DN, Verco L, Carter R, Dzidowski M, Chan PY. Measurement of airborne particle exposure dur- ing simulated tracheal intubation using various proposed aerosol containment devices during the COVID-19 pandemic. Anaesthesia. 2020. 